\title{
CONTROLE DE Hypsipyla grandella ZELLER,1848 (LEPIDOPTERA, PYRALIDAE) EM \\ Swietenia macrophylla KING ATRAVÉS DE SISTEMA AGROFLORESTAL E CONSÓRCIO COM MELIÁCEAS RESISTENTES
}

\author{
Telma F. C. Batista ${ }^{\text {, O Orlando S. Ohashi }}{ }^{1}$, Fabrícia S. Gomes ${ }^{1}$, \\ Paulo R. G. Bittencourt', Rafael C. Ribeiro'
}

${ }^{1}$ Instituto de Ciências Agrárias da Universidade Federal Rural da Amazônia - Ufra. Av. Tancredo Neves n² 2501 CP: 917. CEP: 66.077-530. Montese. Belém-Pa

\section{RESUMO}

O mogno brasileiro Swietenia macrophyla é uma das madeiras brasileiras mais valorizadas no mercado internacional com preço variando de US\$ 1,3 a US\$ $1,6 \mathrm{mil} / \mathrm{m}^{3}$. Entretanto, projetos de plantios comerciais a pleno sol deixaram de ser implantados nas Américas, Central e do Sul, principalmente devido ao ataque da broca Hypsipyla grandella. $\mathrm{O}$ presente trabalho teve o objetivo de avaliar a eficiência de controle da broca $H$. grandella em dois modelos de plantios do mogno, em sistema agroflorestal e consorciado com meliáceas resistentes como Toona ciliata, Khaya ivorensis e Azadirachta indica. Os resultados demonstraram que não ocorreu diferença estatística entre os dois sistemas de plantio, porém $S$. macrophylla plantado com $T$. ciliata propiciou maior eficiência de controle $(48 \%)$, em relação ao ataque da broca, e $10 \%$ e $15 \%$ de efíciência em relação a postura nos consórcios com $K$. ivorensis e $A$. indica, respectivamente.

Palavras-chave: Broca-do-mogno, eficiência de controle, meliáceas resistentes, sistema agroflorestal, consorciamento

\section{CONTROL OF Hypsipyla grandella ZELLER, 1848 (LEPIDOPTERA, PYRALIDAE) IN \\ THE PLANTATION OF Swietenia macrophylla KING THROUGH AGROFOREST SYSTEM AND IN ASSOCIATION WITH RESISTANT MELIACEAE}

\begin{abstract}
The brazilian mahogany Swietenia macrophylla is one of the most expensive woods in the world (US\$ 1,3 to US $\$ 1,6 \mathrm{~m}^{3}$ ). However Projects of commercial plantations in pure stands are not set in Central and South America, mainly due to the shootborer Hypsipyla grandella attack. The efficiency of $S$. macrophylla in the control of $H$. grandella was evaluated in two different models of plantation, in an agroforest system and in association with resistant Meliaceae such as Toona ciliata, Khaya ivorensis and Azadirachta indica. The results showed no statistical difference between these two models of plantation. However $S$. macrophylla in association with $T$. ciliata presented the higher level of control efficiency $(48 \%)$ regarding the shootborer attack and $10 \%$ and $15 \%$ of efficiency on the $H$. grandella egg laying in the association with $K$. ivorensis and $A$. indica, respectively.
\end{abstract}

Key words: Shootborer, control efficiency, resistant meliaceae mahogany, agroforest system, association 


\section{INTRODUÇÃO}

O mogno brasileiro $S$. macrophyla é uma das madeiras mais nobres do mundo, possui um brilho natural que confere à madeira beleza muito especial que aliado às propriedades físicas, é preferido para fabricação de canoas e embarcações, além de ser utilizado atualmente para todas as finalidades de ornamentações de interiores e exteriores de ambientes, sendo por isso aplicado em decorações de requinte, marcenaria de arte, mobiliário, marchetaria, obras de talha etc. (Lamprecht, $1990 \mathrm{e}$ Lorenzi, 1992). Isso contribuíu para a exploração irracional de $S$. macrophylla em reservas naturais, que se concentram no Brasil, especialmente, ao Sul do estado do Pará (Barros et al., 1992 e Baima et al., 2000). Aliado a isso, a falta de fiscalização mais efetiva e permanente, pelos órgãos competentes, favorece para que $S$. macrophylla seja explorada sem a devida reposição ao meio ambiente nativo.

Os sistemas Agroflorestais (SAFs) existem há tempos remotos, estima-se que eram praticados por civilizações antigas na América latina, onde os agricultores desde essa época manejavam os recursos naturais nos quais espécies lenhosas (árvores, arbustos, palmeiras, etc.) são utilizados em associação deliberada com culturas agrícolas e/ou animais na mesma área, de maneira simultânea ou seqüencial temporal, com interações ecológicas e/ou econômicas e significativas entre os componentes (ICRAF, 1983). Enquanto que os consórcios são associações de vegetais, na qual não se tem intuito de planejar quaisquer tipos de interações (ecológicas e/ou econômicas) entre os componentes. Sabe-se, entretanto, que todo sistema agroflorestal é um consórcio, mas, nem todo consórcio é um sistema agroflorestal.

Neste sentido, pesquisas sobre alternativas de manejo de plantas de $S$. macrophylla devem ser incrementadas, de maneira que possam ser utilizadas mais expressivamente no controle da broca, com ênfase ao desenvolvimento sustentável e ecológico da região Amazônica.

O objetivo deste trabalho foi avaliar a eficácia de controle da broca $H$. grandella em dois diferentes tipos de plantios : sistema agroflorestal e consórcio com meliáceas resistentes.

\section{MATERIAL E MÉTODOS}

O experimento foi realizado, no ano de 2003, no município de Aurora do Pará a $220 \mathrm{~km}$ de Belém-PA. O plantio no campo das espécies florestais foi em parcelas de 15 x $15 \mathrm{~m}$ e espaçamento de $3 \times 1,5 \mathrm{~m}$. Foi semeado posteriormente o feijão caupi nas entre-linhas, somente nas subparcelas sorteadas com sistema agroflorestal. Após a colheita do feijão toda a biomassa foi incorporada no solo. A plantio de feijão teve o objetivo de fixar maiores quantidades de nitrogênio no solo. $\mathrm{O}$ delineamento foi em blocos casualizados com parcelas subdivididas e 4 repetições. A variável nas parcelas foram às espécies florestais e nas subparcelas o caupi. Cada parcela experimental constituiu-se de duas subparcelas com 18 plantas cada.

Os tratamentos nas unidades experimentais constaram de: $S$. macrophylla $+T$. ciliata; $S$. macrophlyla $+K$. ivorensis; S. macrophylla + A. indica; S. macrophylla, $T$. ciliata; $K$. ivorensis e $A$. indica.

Os parâmetros avaliados foram: número de plantas de $S$. macrophyla atacadas e número de ovos de $H$. grandella presentes nas plantas. Os dados obtidos foram submetidos à análise de variância e as médias comparadas pelo teste Duncan (5\%). As médias do número de ovos foram corrigidas pela fórmula $\sqrt{ } \mathrm{x}+0,5$. O sistema de análise estatística utilizado foi o SAEG de Ribeiro Júnior \& Ivo (2001).

A percentagem de eficiência de controle foi calculada pela fórmula de Abbot 
T descrita por Nakano et al. (1981), que é \% $\mathrm{Ef}=(\mathrm{T}-\mathrm{I}) / \mathrm{T}_{-} \times 100$, onde $\mathrm{T}=\mathrm{n}^{\circ}$ de insetos na testemunha ( $S$. macrophyla) e o $\mathrm{I}=\mathrm{n}^{\circ}$ de insetos no tratamento.

\section{RESULTADOS E DISCUSSÃO}

Os resultados demonstraram que não houve diferença estatística entre os dois modelos de plantios avaliados para $o$ parâmetro ataque de $H$. grandella ás plantas de meliáceas (Tabela 1).

Tabela 1. Análise de variância do número médio de ataques por Hypsipyla grandella Zeller sobre o mogno brasileiro Swietenia macrophylla King, em plantio homogêneo e consorciado com meliáceas resistentes e caupi

\begin{tabular}{lcccc}
\hline Fontes de Variação & GL & $\begin{array}{c}\text { Soma do } \\
\text { Quadrado }\end{array}$ & Quadrado Médio & $f$ \\
\hline Total & 31 & 28.17969 & - & - \\
Total de redução & 19 & 25.72656 & 1.354030 & 6.6 \\
Bloco & 3 & 0.303125 & 0.1067708 & 0.6 \\
Planta & 3 & 23.28906 & 7.763021 & $46.3^{* *}$ \\
Erro (A) & 9 & 1.507812 & 0.1675347 & - \\
Caupi & 1 & 0.7812500 & 0.7812500 & 0.0 \\
Planta x Caupi & 3 & 0.6015625 & 0.2005208 & 0.9 \\
Resíduo & 32 & 2.453125 & 0.2044271 & - \\
\hline
\end{tabular}

Número de dados: 32

Média Geral: 2,9219

Coef. Variação: 15.474

**Altamente significativo ao nível de $1 \%$ de probabilidade

Os tratamentos homogêneos de $T$. ciliata, $K$. ivorensis e $A$. indica, não foram atacados pela broca, em quaisquer dos dois modelos de plantio. Esses resultados indicam que tais plantas propiciaram de $100 \%$ de controle sobre $H$. grandella, enquanto que as plantas de $S$. macrophylla em consórcio e em SAF com $K$. ivorensis, $A$. indica e $T$. ciliata e mais o caupi propiciaram controle de $39 \%$, $46 \%$ e $48 \%$, respectivamente (Figura 1 ).

Apesar das plantas de $S$. macrophylla, nos dois modelos de plantio, terem obtido eficiência regular de controle, esses resultados são importantes quando se visualiza futuros trabalhos de manejo integrado da broca-do-mogno, tendo em vista que o plantio consorciado de $S$. macrophylla com meliáceas resistentes é compatível com qualquer outra medida de controle. Segundo Costa (2000), o consórcio de $S$. macrophylla com $T$. ciliata associado à cola stick, colocada na parte intermediária do broto novo, propiciou eficiência de controle de $88 \%$, porque a broca fica aderida à cola. Entretanto, Ohashi et al. (2002), utilizando esse consórcio associado a Colacid (cola stick + inseticida) obtiveram eficiência de até $100 \%$.

Esses resultados são importantes porque foi observado que com $S$. macrophylla em plantios homogeneos apresentaram em média 4 ataques/planta/ano, ou seja, o mogno brasileiro foi mais suscetível ao ataque da broca, do que quando consorciado e em SAF, que tiveram de 2 a 3 ataques/planta/ano (Figura 2). Isso demonstra que ocorreu controle da broca nas plantas de $S$. macrophylla nos tratamentos utilizados, mesmo sabendo-se que o consorciamento e o SAF estão no início de estabelecimento, período em que o 


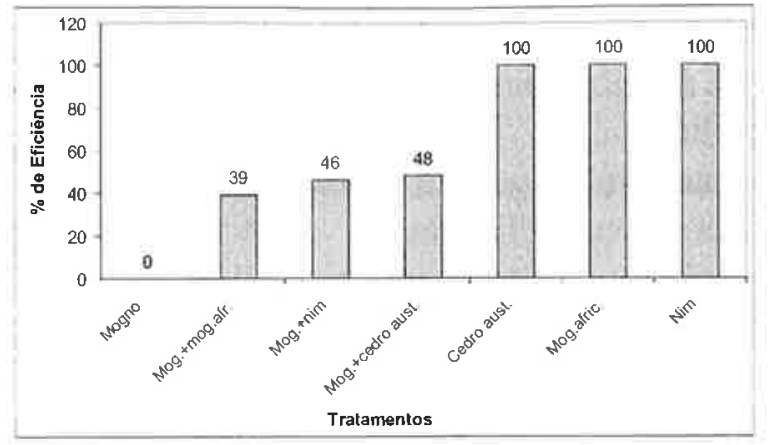

Figura 1. Percentual de eficiência de controle das plantas de mogno brasileiro $(S$. macrophylla) e meliáceas resistentes (cedro, nim e mogno africano) em relação aos ataques de Hypsipyla grandella em sistemas consorciado e em SAF no município de Aurora do Pará-PA durante o ano de 2003.

experimento foi avaliado. Provavelmente, essa efíciência aumentará à medida em que as plantas de $S$. macrophylla cresçam e se estabeleçam no local, pois de acordo com Hilje e Cornelius (2001) os três primeiros anos constituem um período crítico ao ataque da broca e quando o $S$. macrophylla atinge em média $6 \mathrm{~m}$ de altura os danos são menores porque a planta apresenta maior capacidade de recuperação.

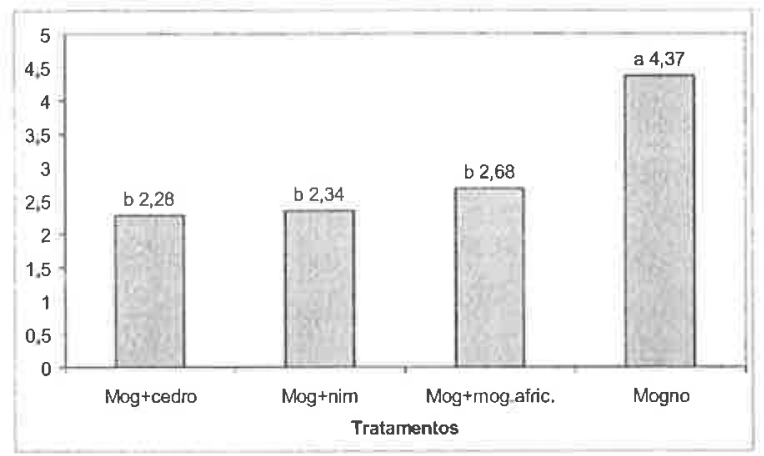

Figura 2. Número médio de ataques por Hypsipyla grandella em plantas de mogno brasileiro (S. macrophylla) consorciado e em SAF com meliáceas resistentes (cedro, nim e mogno africano) no município de Aurora do Pará durante o ano de 2003. Médias precedidas de letras distintas diferem entre si pelo Teste de Duncan (5\%).
Quanto à postura, a eficiência de controle exercida pelo plantio de $T$. ciliata plantada com S. macrophylla, nos dois modelos de plantio, foi de $21 \%$, enquanto que nos tratamentos de $S$. macrophylla com $A$. indica e $S$. macrophylla com $K$. ivorensis foram de $15 \%$ e $10 \%$, respectivamente (Figura 3). Esses resultados concordam com os observados por Costa (2000), que verificou maior postura no plantio homogêneo de $S$. macrophylla do que quando consorciado com $T$. ciliata.

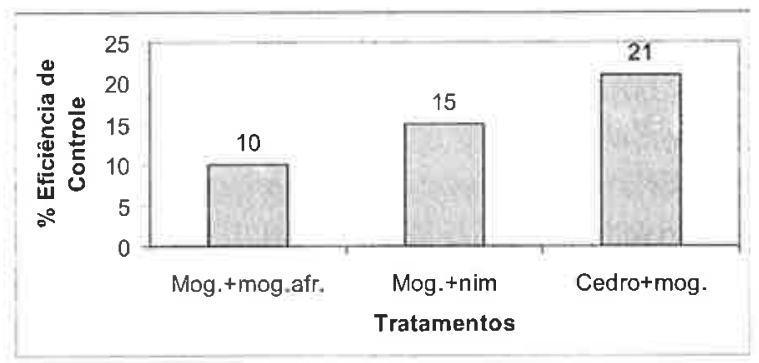

Figura 3. Percentual de eficiência de controle das plantas do Swietenia macrophyla e do Toona ciliata em relação as posturas de Hypsipyla grandella em sistemas consorciado e em SAF com meliáceas resistentes (cedro, nim e mogno africano) no município de Aurora do Pará

Apesar de S. macrophylla plantado com $T$. ciliata atingir maior índice de postura de $H$. grandella em relação a $S$. macrophylla em plantio homogeneo, foi nesse consórcio que as plantas de $S$. macrophylla obtiveram $48 \%$ de eficiência de controle em relação aos ataques (Figura 1 e Tabela 2), sendo o maior índice entre os tratamentos avaliados. Além disso, as plantas de $S$. macrophylla nos consórcios e no SAF apresentaram médias de apenas 2 a 3 ataques/planta/ano, enquanto que no plantio solteiro dessa espécie ocorreram cerca de 4 ataques/planta/ano (Figura 2).

S. macrophylla plantado com $T$. ciliata foi o tratamento que mais atraiu posturas de $H$. grandella, com média de cerca de 14 ovos/100 plantas durante 0 
primeiro mês de avaliação (Tabela 2), ou seja, são plantas de espécies diferentes, que apesar de serem da mesma família, que consorciadas intensificaram ainda mais o potencial de atração para oviposição de $H$. grandella em relação aos outros consórcios avaliados neste trabalho. Entretanto, esse mesmo tratamento alcançou em média $21 \%$ de eficiência de controle sobre a postura de $H$. grandella, durante os três meses de avaliação. Esse resultado é importante porque demonstrou que $T$. ciliata atrai a mariposa para oviposição e mata a broca por antibiose, conforme demonstraram trabalhos de laboratório de Grijpma, Roberts (1973, 1975), Oiano Neto (2000), Costa (2000) e Sarmento Júnior (2001). Nesse caso, vários ovos de $H$. grandella deixaram de ser depositados sobre $S$. macrophylla, que é suscetível a broca e, conseqüentemente, diminuindo a pressão de ataque sobre o mogno brasileiro.

A preferência de oviposição de $H$. grandella sobre $T$. ciliata também foi observada no trabalho de Costa (2000) porque, provavelmente, as substâncias voláteis que atraem as fêmeas de $H$. grandella, podem ser mais estimulantes nessa planta do que em relação ao mogno brasileiro, conforme demonstrou Oiano Neto (2000).

Esses resultados concordam com os obtidos por Costa (2000) que avaliou o consorciamento de $S$. macrophylla e $T$. ciliata em diferentes espaçamentos e obteve médias de eficiência de controle de postura de $H$. grandella para $T$. ciliata que variaram de $82 \%$ a $87 \%$.

O segundo tratamento mais preferido para oviposição foi a planta de mogno brasileiro consorciada com o mogno africano e mogno brasileiro com o nim, os quais não diferiram estatisticamente do mogno em plantio puro, com médias que variaram de 6 a 9 ovos/100 plantas (Tabela 2). Entretanto, nas plantas de nim e mogno africano não foi observada a presença de nenhum ovo, por isso não foram atacadas $\mathrm{e}$, portanto, consideradas resistentes pela não preferência para oviposição por $H$. grandella.

Tabela 2. Comparação das médias de postura de Hypsipyla grandella transformadas por $\sqrt{(x+0,5)}$ sobre plantas de Swietenia macrophylla e Toona ciliata consorciados e em SAF com meliáceas resistentes (cedro, nim e mogno africano) no município de Aurora do Pará-PA durante os meses de fevereiro, março e abril de 2003.

\begin{tabular}{lrrrc}
\hline Mês & Fev. & Março & Abril & $\begin{array}{c}\text { Média Ovos } \\
\text { Trimestre }\end{array}$ \\
\hline Tratamentos* & & & & \\
\hline - S. macrophylla + T. ciliata & $5,20 \mathrm{~b}$ & $6,68 \mathrm{~b}$ & $9,62 \mathrm{a}$ & $7,16 \mathrm{bc}$ \\
- $T$. ciliata + K. ivorensis & $14,96 \mathrm{a}$ & $3,27 \mathrm{~b}$ & $10,18 \mathrm{a}$ & $9,47 \mathrm{~b}$ \\
- S. macrophylla + K. ivorensis & $7,65 \mathrm{~b}$ & $3,90 \mathrm{~b}$ & $9,53 \mathrm{a}$ & $7,03 \mathrm{bc}$ \\
- S. macrophylla + A. indica & $3,95 \mathrm{~b}$ & $4,58 \mathrm{~b}$ & $9,60 \mathrm{a}$ & $6,04 \mathrm{c}$ \\
- S. macrophylla & $6,80 \mathrm{~b}$ & $3,37 \mathrm{~b}$ & $11,10 \mathrm{a}$ & $7,09 \mathrm{bc}$ \\
- - T. ciliata & $13,36 \mathrm{a}$ & $12,35 \mathrm{a}$ & $10,37 \mathrm{a}$ & $12,03 \mathrm{a}$ \\
\hline
\end{tabular}

*Médias de ovos de Hypsipyla grandella a cada 100 plantas seguidas por letras distintas na vertical diferem entre si pelo Teste de Duncan 5\%. Nos consórcios considerar média de ovos para a primeira planta.

Em se tratando de controle alternativo, como planta armadilha ou planta isca, dentre as meliáceas resistentes avaliadas a $T$. ciliata foi planta que alcançou melhor desempenho, inclusive como barreira biológica para $H$. grandella, tanto na eficiência de controle de ataques, que foi de $100 \%$ como na eficiência de postura que 
ficou em média de $21 \%$. A opção de consorciamento do $S$. macrophylla com $T$. ciliata, em áreas de reflorestamento, pode ser uma das práticas de manejo de controle de $H$. grandella mais desejáveis em relação ao uso de produtos químicos, pois se sabe que são mais caros, aumentam a poluição do meio ambiente e dos custos de manutenção da área de plantio e ainda podem causar desequilíbrios ambientais se não forem utilizados corretamente e indiscriminadamente.

\section{REFERÊNCIAS BIBLIOGRÁFICAS}

BAIMA, A. M. V.; SILVA, J. N. M.; JENNINGS, S. B., ALMEIDA, W. C.; LOPES, J. C. A.; CARVALHO, J. O. P.; MIRANDA, I. L.; SOARES, M. H. M.; FERREIRA, F. N., 2000. Estrutura de duas florestas exploradas com ocorrência de mogno (Swietenia macrophylla King) nas regiões de Marabá e Rio Maria do Pará. Embrapa Amazônia Oriental. 4p. 2000 (Comum. Tec. no 49).

BARROS, P. L. C.; QUEIROZ, W. T.; SILVA, J. N. M.; OLIVEIRA, F.; FILHO, B. P. C.; TEREZO, F. E. M.; VILACORTA, A., 1992. Reservas naturais e artificiais de Swietenia macrophylla King na Amazônia brasileira: numa perspectiva de conservação: Faculdade de Ciências Agrárias do Pará. Belém. 56 p.

COSTA, M. S. S., 2000. Controle de Hypsipyla grandella (Broca do mogno) utilizando a planta resistente Toona ciliata Roem (Cedro australiano) e os métodos mecânico e cultural no plantio de Swietenia macrophylla (Mogno). FCAP, Dissertação de Mestrado, 52 p.

GRIJPMA, P; ROBERTS, S. C. On the resistance of Toona ciliata $\mathrm{M}$ J. Roem. Var. Australis (F. V. M.) Procedings se. N.17. p. 1-14. In: Symposium of
Integrated Control of Hypsipyla. Turrialba, 1973.

GRIJPMA, P; ROBERTS, S. C. Studies on the shootborer Hypsipyla grandella (Zeller) (Lep. Pyralidae). XVII. Biological and chemical screening for the basis of resistance of Toona ciliata M J. Roem. Var. Australis. Turrialba, n.25. p.152-59. 1975.

LAMPRECHT, H., 1990. Silvicultura nos trópicos. Ecossistemas florestais $\mathrm{e}$ respectivas espécies arbóreas Possibilidades e métodos de aproveitamento sustentado. Cooperação Técnica. República Federal da Alemanha. Eschborn. 342 p. HILJE, L.; CORNELIUS, J., 2001. Es inmanejable Hypsipyla grandella como plaga florestal. Turrialba. CATIE. Hoja Técnica. n.18. 4 p.

ICRAF. 1983. International Council foi Research an Agroforesty. Alternatives to Slash \& Burn: Global Strategy, Martinique, 107p.

LORENZI, H., 1992. Árvores do Brasil: Manual de identificação e cultivo de plantas arbóreas nativas do Brasil. Editora Plantarum. Nova Odessa, SP. p.241.

NAKANO, O.; SILVEIRA NETO, S.; ZUCCHI, R. A., 1981. Entomologia econômica. São Paulo: Livro ceres. $314 \mathrm{p}$.

OHASHI, O. S.; SILVA, J. N. M.; SILVA, M. F. G. F.; COSTA, M. S. S.; SARMENTO JÚNIOR, R. G.; SANTOS, E. B.; ALVES, M. Z. N.; PESSOA, A. M. C.; SILVA, T. C. O.; BITTENCOURT, P. R. G.; BARBOSA, T. C.; SANTOS, T. M., 2002. Manejo Integrado da broca do mogno Hypsipyla grandella Zeller (Lep. Pyralidae). In: POLTRONIERE, L. S.; TRINDADE, D. R. Manejo integrado das principais pragas $e$ doenças de cultivos amazônicos. 
Belém: Embrapa Amazônia Oriental. cap.VII. p.91-120.

OIANO NETO, J. O., 2000. Estudo fitoquímico de Toona ciliata: uma contribuição à quimiossistemática do gênero e à ecologia da interação Hypsipyla-Meliaceae. São Carlos: UFScar, 287 p. Tese de Doutorado.

RIBEIRO JÚNIOR.; IVO, J., 2001. Análises estatísticas no SAEG. Viçosa: UFV. 301p.
SARMENTO JÚNIOR, R. G. Biologia de Hypsipyla grandella (Zeller, 1848) e avaliação da resistência de Swietenia macrophylla king., Cedrela odorata L., Toona ciliata Roem e Toona sp. à broca das meliáceas em laboratório. FACP, dissertação de mestrado, $61 \mathrm{p}$. 2001. 